\title{
Social Recommendation Based on the Analysis of Interest and Follow Influences
}

\author{
Chin-Hui Lai ${ }^{\mathrm{a},}{ }^{*}$, Duen-Ren Liu ${ }^{\mathrm{b}}$, Chi-Hao Yeh ${ }^{\mathrm{b}}$ \\ a Department of Information Management, Chung Yuan Christian University, Taoyuan 320, Taiwan \\ b Institute of Information Management, National Chiao Tung University, Hsinchu 300, Taiwan \\ *Corresponding Author: chlai@cycu.edu.tw
}

\begin{abstract}
With its flourishing development of Web 2.0, people not only passively receive the information, but actively share the information with others by web 2.0 technology. Yet, for people, the information overload problem leads difficulty in filtering the explosive information and identifying the needed information. To solve the problem, the recommender systems which make recommendations based on users' preferences or the contents of items are the widely utilized solutions. However, the interest influence, follow influence and personalized weights of influences may be the important factors for making recommendations. Besides, the related researches do not consider the review influence and the time factor in recommendation. In this work, the novel recommendation method based on two types of influences, including the interest influence and follow influence, and personalized weights for each influence, is proposed for recommending products in a cosmetic-sharing website. The experimental results show the proposed method improves the performance of recommendation.
\end{abstract}

Keywords: Social Network, Recommender System, Influence Analysis, Social Recommendation.

\section{Introduction}

Web 2.0 generates the new era of internet communication for people. Because of the state-of-the-art Web technology, people have various ways to communicate with each other anytime anywhere. A web 2.0 site allows users to interact and collaborate with other users in a social-related way. Thus, people not only receive the information from the Web, but actively share the user-generated content with others by using web 2.0 services. However, information explosion leads to the information overload problem. People have difficulties in finding their needed information. To solve the problem, the recommender system is a solution to filter and provide suitable information or items, such as videos, tags, and webpages, to users based on their preferences.

In this work, a novel recommendation method based on interest influence and follow influence is proposed. Interest influence means that users' buying behavior is influenced by their similar users with similar interest. It is measured by the number of the same products which users are influenced to get interested or buy by their similar users. Follow influence means that users are affected by their followees whom users are interested in and followed. The follow influence consists of purchase-follow influence, interaction strength and review influence. The purchase-follow influence is that users are influenced by their followees to buy the same products their followees have bought. The interaction strength is used to find the followees whom users interact frequently. Such followees may have much more influence than other followees. The review influence means that users may be influenced by other users' product reviews. Because of the power of word-of-mouth marketing, such kind of influence may affect users' buying decisions more greatly than the interest influence.

Additionally, the interest and follow influence are adapted to each user by giving the personalized weights, since the influences have different impact on the user. Finally, the interest influence and follow influence are incorporated in the proposed recommendation method to recommend products for the target users. Such influences can improve the recommendation quality when the recommendation method is used on a social network. To evaluate the proposed mothod, we conduct several 
experiments based on the collected dataset from a popular social network website in Taiwan- Urcosme ${ }^{1}$. From the experimental results, our proposed methods can indeed improve the performance of the recommendation. Based on the degree of interactions between a user and his/ her followees, the followees are more influential in reaching buying decisions of the user than his/ her similar users.

The remainder of this paper is organized as follows. Section 2 introduces the related works. In Section 3, the proposed recommendation methods based on interest and follow influences are discussed respectively. Section 4 shows the experimental results and evaluations of the proposed methods. Section 5 presents the conclusions of this work.

\section{Related Work}

The trust-based social recommender systems can calculate the confidence between users by using a trust metric to quantify the degree of the trust ${ }^{(1)}$. As trust is applied in social networks, it provides more functions for the expansion of Internet intelligence. For example, Epinions.com allows users to express their trust opinions by adding a reviewer into their trust list or block list according to whether or not the reviewer's reviews are valuable, and then takes advantage of trust to balance the collaborative recommender system's defects ${ }^{(2)}$. Based on a trust network, the influential capability of online reviewers can be evaluated and then recommend appropriate reviewers to support the word-of-mouth marketing ${ }^{(3)}$.

Social influence means that the behavior of users is influenced by their friends on a social network. Some studies have measured social influence from blogs, tweets and tags by mining users' behavior to make recommendations for a target user. Cui et al. ${ }^{(4)}$ evaluated the influence of a user by the number of his/her friends who click the shared web posts. The influence of a user has also been calculated by the ratio of the number of items a target user received to the number of items a recommender system recommended ${ }^{(5)}$. In addition, a mathematical programming model $^{(6)}$ is proposed and applied to the influence networking sites for identifying user groups with large joint influential power. Based on the social influence on a social network, information can be disseminated quickly through those influential users, and the items recommended by those influential users are easily accepted by others.

\footnotetext{
1 http://www.urcosme.com/
}

\section{Recommendation of Influence Analysis}

\subsection{Overview}

The overview of our proposed recommendation method is shown in Fig. 1. First, a dataset is collected from a social network website, and then users' personal profiles are generated from the collected dataset. In the phase of influence analysis, interest influence and follow influence are defined and analyzed. Interest influence means that a user may be affected by other users who have similar interests or preference. Based on users' interactions on a social network, the influenced users who have similar preferences can be identified for each user. Additionally, users may have connection with others by making friends or follow other users' behavior, e.g. posting reviews. When a user follows other users, the user is a follower of these users and they are the followees of the user. In such following relationship, three main factors are considered in measuring the follow influence: purchase-follow influence, interaction strength, and review influence. Because these influences may have different impact on users on a social networking website, the personalized weights represents the importance of these two influences. Finally, the scores of the interest and follow influences are combined by using the personalized weights to create a recommendation list. Based on the list, top- $k$ products with high scores are recommended to a target user.

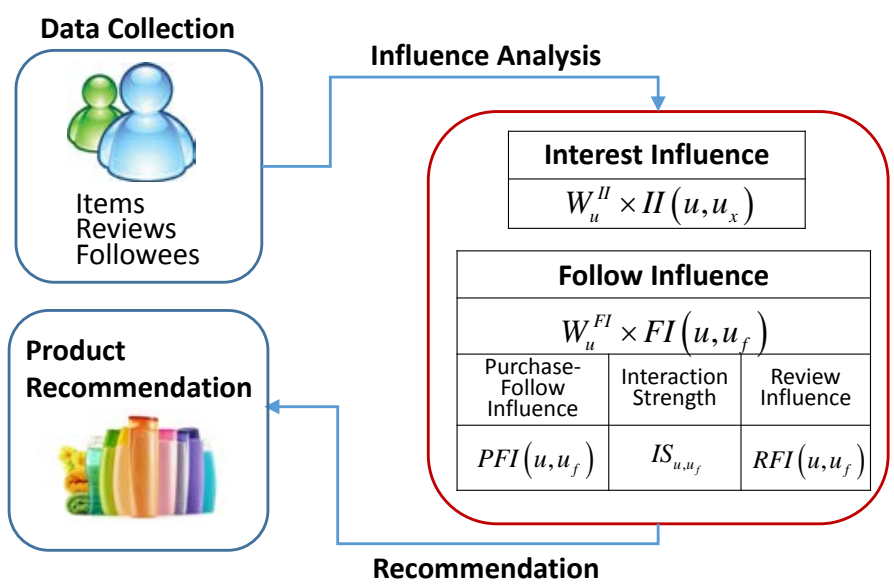

Fig. 1. The proposed recommendation model.

\subsection{Interest Influence}

The interest influence means that users are influenced by users with similar preferences. According to users' bought and wish lists, two users who add the same products into their bought and wish lists or like the same users' opinions have similar preferences. Thus, the interest 
influence is measured by the number of particular products added into a user's bought and wish list after his/ her similar users have added these products. To identify similar users for each user, Jaccard coefficient is used to evaluate the similarity between two users, as defined in Eq.(1).

$$
\operatorname{Jsim}\left(u, u_{x}\right)=\frac{\left|I_{u} \cap I_{u_{x}}\right|}{\left|I_{u} \cup I_{u_{x}}\right|}
$$

where $\operatorname{Jsim}\left(u, u_{x}\right)$ is the Jaccard similarity between user $u$ and user $u_{x} ; I_{u}$ and $I_{u_{x}}$ are the bought and wish lists of user $u$ and $u_{x}$ respectively. The bought list consists of a user's previously bought products, while the wish list consists of products that a user wants to buy. Based on Jaccard similarities, i.e., Jsim, top- $K$ users with high similarities are chosen as similar users of a target user $u$.

The interest influence is derived from the perspectives of influential user (user $u_{x}$ ) and influenced user (user $u$ ). To obtain reliable values of interest influence for users, two perspectives of influenced user and influential user are combined linearly by using a parameter $\alpha$ as a weight in computing the interest influence, as shown in Eq. (2).

$$
I I\left(u, u_{\chi}\right)=\alpha \times \frac{\left|I_{u_{x} \rightarrow u}\right|}{\left|I_{u}\right|}+(1-\alpha) \times \frac{\left|I_{u_{x} \rightarrow u}\right|}{\left|I_{u_{x}}\right|}
$$

where $\left|I_{u_{x} \rightarrow u}\right|$ is the number of products in the bought and wish lists added by user $u$ after user $u_{x}$ has added them, $\left|I_{u}\right|$ is the number of products in user $u$ 's bought and wish lists, $\left|I_{u}\right|$ is the number of products in user $u_{x}$ 's bought and wish lists and $\alpha$ is a parameter which ranges from 0 to 1 . If $\alpha$ equals to 1 , the interest influence of user $u_{x}$ on user $u$ is totally derived from the perspective of influenced user $u$. On the other hand, if $\alpha$ equals to 0 , the interest influence of user $u_{x}$ on user $u$ is totally derived from the perspective of influential user $u_{x}$. When $\alpha$ equals to 1 or 0 , the interest influence is only derived from only one single perspective.

The interest influence may have different degrees of impact on users. The personalized weight of interest influence for each user represents the impact degree of the interest influence. Such personalized weight is calculated according to the number of common items that both a user and his/her similar users have, as defined in Eq. (3).

$$
W_{u}^{I I}=\frac{\left|\bigcup_{u_{x} \in \mathrm{NBR}_{u}} I_{u_{x} \rightarrow u}\right|}{\left|I_{u}\right|}
$$

where $W_{u}^{\text {II }}$ is a personalized weight of interest influence to user $u ; N B R_{u}$ is a set of top- $K$ similar users of user $u ;\left|\bigcup_{u \in N B R} I_{u_{x} \rightarrow u}\right|$ is the number of a set of products added by user $" u$ into the bought and wish lists after the similar user $u_{x}$ have added them. For a user, a high weight of interest influence means that the interest influence largely impact on the user.

\subsection{Follow Influence}

From users' interaction, users may be influenced by their friends or followees. The follow influence is composed of purchase-follow influence, interaction strength, and review influence. In the following subsections, the computation detail of the follow influence is discussed.

\subsubsection{Purchase-Follow Influence}

Generally, users' purchasing behavior may be affected by their followees. User $u$ follows user $u_{f}$ to buy or add the same products in his/her bought and wish lists, after user $u_{f}$ has add the products to his/her list; or after user $u_{f}$ has written the review of the products. That is, users can select interested products into their own bought and wish lists by tracing the bought and wish lists or reviews of their followees. Such user behavior is regarded as purchase-follow influence. Similar to the concept of interest influence mentioned in section 3.2, two perspectives of the purchase-follow influence are taken into account during the computation. To completely represent the purchase-follow influence, the parameter $\beta$ ranging from 0 to 1 is used to combine the scores of the purchase-follow influence between two users from the perspectives of both influenced and influential users respectively, as defined in Eq. (4) .

$$
\operatorname{PFI}\left(u, u_{f}\right)=\beta \times \frac{\sum_{i \in I_{u_{f} \rightarrow \mu}} t f_{i}}{\left|I_{u}\right|}+(1-\beta) \times \frac{\sum_{i \in L_{u_{f} \rightarrow \mu}} t f_{i}}{\left|I_{u_{f}}\right|}
$$

where $\operatorname{PFI}\left(u, u_{f}\right)$ is user $u$ 's purchase-follow influence on user $u_{f} . u_{f}$ is a follower of user $u$, while user $u$ is a followee of user $u_{f} \cdot I_{u_{f} \rightarrow u}$ is a set of products, which user $u$ selected into the bought and wish lists after user $u_{f}$ has added; or after user $u_{f}$ has written the reviews about these products. $\sum_{i \in I_{u, \ldots+}} t f_{i}$ is the sum of time factor score on each purchase-follow link from user $u_{f}$ to user $u$ for the products in $I_{u_{f} \rightarrow u}$.

The time factor, as defined in Eq. (5), is considered in calculating the purchase-follow influence since the link of 
purchase-follow relationship between two users occurs at different time. The value of the time factor is used to represent the importance of a link.

$$
t f_{d}=e^{-\tau\left(\mathrm{t}_{\text {now }}-\mathrm{t}_{d}\right)}
$$

where $t f_{d}$ is the time factor, $t_{\text {now }}$ is the current time, $t_{d}$ is the time that user $u$ adds the product $d$ into his/her bought and wish list, and the parameter $\tau$ is used to evaluate the impact of time factor.

\subsubsection{Interaction Strength}

The interaction strength between users and their followees are analyzed to find close followees for the user. The opinions and preferences of those followees may influence greatly the user. Therefore, the interaction between users and their followees in a social network can be regarded as a type of edge. Based on the interaction edges between two users, their interaction strength can be calculated by using Eq.(6).

$$
I S_{u, u_{f}}=\sum_{u_{f} \in F_{u}, e d g e s \in\left(u, u_{f}\right)} e_{u, u_{f}} \times t_{u, u_{f}}
$$

where $I S_{u, u}$ is the interaction strength of user $u$ and user $u_{f}$ who is the followee of user $u . F_{u}$ is a set of followees of user $u$; $t_{u, u_{f}}$ is the time factor calculated by using Eq. (5). $e_{u, u_{f}}$ means a weight of an edge between user $u$ and user $u_{f}$ depending on their interaction behavior. If a user highly interacts with his/ her followees on a specific type of interaction, the weight of such type of interaction is large.

\subsubsection{Review Influence}

Review influence is the influence from the followees having the review of products. The review influence derived from both the influenced users and influential users is defined in Eq.(7).

$$
\operatorname{RFI}\left(u, u_{f}\right)=\gamma \times \frac{\sum_{i \in I R_{u_{f} \rightarrow u}} t f_{i}}{\left|I_{u}\right|}+(1-\gamma) \times \frac{\sum_{i \in I R_{u_{f} \rightarrow u}} t f_{i}}{\left|I_{u_{f}}\right|}
$$

where $\sum_{i \in I R_{u_{f} \rightarrow u}} t f_{i}$ is the summation of the time factor scores of links which user $u$ adds the same products in the list after user $u_{f}$ has written the reviews of these products. The parameter $\gamma$, ranging from 0 to 1 , is used to combine the review influence from the both perspectives of the influenced users and the influential users.

The buying behavior of each user is affected by different followees. Some are influenced much by their followees, and the others may not. To adapt each user, the weight of follow influence for a user is defined in Eq.(8).

$$
W_{u}^{F I}=\frac{\left|\bigcup_{u_{f} \in F_{u}} I_{u_{f} \rightarrow u}\right|}{\left|I_{u}\right|}
$$

where $W_{u}^{F I}$ is the weight of follow influence for user $u$; $F_{u}$ is the set of Top- $K$ followees with high follow influence of user $u ;\left|\bigcup_{u_{\epsilon} \in E_{u}} I_{u_{t} \rightarrow u}\right|$ is the set of products added by user $u$ after the followees $u_{f}$ have added them. Large value of the weight means that a user greatly influenced by his/ her followees.

\subsection{Recommendation}

A recommendation method is proposed, which is a user-based collaborative filtering with interest influence and follow influence and personalized weight. According to the influence analysis, the interest influence and follow influence are linearly combined as an influence score by using the weights of the influences respectively. Finally, we choose the Top- $N$ products with high influence scores to make recommendation for a target user. To predict a score for a product, two types of influence, i.e., interest and follow influence, and the weights of these influences are combined in our recommendation method. The prediction formula is defined as Eq.(9).

$$
\begin{aligned}
& p_{u, i}=W_{u}^{I I} \times \sum_{u_{x} \in N B R_{u}, i \in I_{u_{x}}} I I\left(u, u_{x}\right)+W_{u}^{F I} \times \sum_{u_{f} \in F_{u}, i \in I_{u_{f}}} F I\left(u, u_{f}\right) \\
& \left\{\begin{array}{l}
F I\left(u, u_{f}\right)=P F I\left(u, u_{f}\right) \times\left(1+I S_{u, u_{f}}\right) \times\left(1+\operatorname{RFI}\left(u, u_{f}\right)\right) \\
F I\left(u, u_{f}\right)=P F I\left(u, u_{f}\right) \times\left(1+I S_{u, u_{f}}\right)
\end{array}\right. \\
& \text { if }\left\{\begin{array}{l}
\operatorname{review}_{i} \in u_{f} \\
\operatorname{review}_{i} \notin u_{f}
\end{array}\right. \\
& \text { where } \sum_{u_{x} \in N B R_{u}, i \in I_{u_{x}}} I I\left(u, u_{x}\right)
\end{aligned}
$$

interest influence between a target user $u$ and his/ her Top- $K$ similar users $N B R_{u}$ who have added the recommended product $i$ into their bought or wish lists. $\sum_{u_{f} \in F_{u}, i \in I_{u_{f}}} F I\left(u, u_{f}\right)$

is the summation of the follow influence between a target user $u$ and his/her Top- $K$ followees $F_{u}$ who have added the recommended product into their bought or wish lists. In follow influence, there are two type computations: with or without the review influence. If the followees do not have 
the review of the recommended product $i$, we only use the value from the purchas-follow influence and interaction strength in the computation. If the followees have the review of the recommended product $i$, we add the review influence into the above computation.

\section{Experiment and Evaluations}

\subsection{Experiment setup}

In our experiment, we collect a dataset from a famous social network website, called Urcosme, in Taiwan. This website, focusing on cosmetic, allows users to upload their reviews and the information of cosmetics, share their bought and wish lists, follow the users who are interested in, and so on. Our dataset is composed of 6,139 users, 29,009 reviews of particular cosmetics, and 34,564 particular cosmetics in these users' bought and wish lists. On average, each cosmetic has bought by 3 users and each user has 60 cosmetics in his/her bought and wish lists. The dataset is divided into $70 \%$ for training, $10 \%$ for parameter determination, and $20 \%$ for testing. The training data is utilized in our proposed methods to create and analyze the personal profiles and then generate the recommendation lists. Finally, the testing data is used to evaluate the performance of recommendation methods.

In this work, the user-based CF methods based on the combination of the interest influence and follow influence by using personalized weights are applied to cosmetics recommendation for users. The compared methods in the experiments are listed as follows.

- User-based Collaborative Filtering (UCF): The Jaccard similarity, as defined in Eq.(2), is used in traditional user-based CF method to filter and make recommendations.

- Interest Influence Filtering (II): recommendation only with the score of the interest influence, as defined in Eq.(2).

- Follow Influence Filtering (FI): recommendation only with the score of the follow influence which consists of purchase-follow influence, interaction strength, and review influence, as defined in Eq.(4) , Eq.(6) , and Eq.(7).

- Purchase-Follow Influence (PFI) Filtering, recommendation only with the score of the purchase-follow influence as defined in Eq.(4) .

- Interaction Strength (IS) Filtering, recommendation only with the score of the interaction strength as defined in Eq.(6)

- Purchase-Follow Influence and Interaction Strength (PFI+IS) Filtering, recommendation with the score of the purchase-follow influence and interaction strength as defined in Eq. (4) and Eq.(6).

- Purchase-Follow Influence and Review Influence (PFI+RFI) Filtering, recommendation with the score of the purchase-follow influence and review influence as defined in Eq.(4) and Eq.(7).

- Interest Influence and Follow Influence (II+FI) Filtering: recommendation with the combination of the interest influence and follow influence and personalized weights, as defined in Eq.(9).

\subsection{Evaluation metrics}

To compare the accuracy of the recommendation methods, we utilize the precision and recall metrics. They are used to evaluate the performance for recommendation system popularly ${ }^{(7)}$. Precision is computed by the correctly recommended cosmetics to users and the number of the recommended cosmetics, and it is defined as in Eq.(10). Recall is computed by the correctly recommended cosmetics to users and the number of the cosmetics in the lists, and it is defined as in Eq.(11).

$$
\begin{aligned}
& \text { precision }=\frac{\text { Number of the correctly recommended cosmetics }}{\text { Number of the recommended cosmetics }} \\
& \text { recall }=\frac{\text { Number of the correctly recommended cosmetics }}{\text { Number of the cosmetics in the bought and wish lists }}
\end{aligned}
$$

The number of the cosmetics in the lists means, for the users, they have already bought or been interested in the cosmetics, and added them into their bought list or wish list. Even though these two metrics are simple to use, it would face the problem that if the size of data set increases, the performance of two metrics also improves. Therefore, the F1-metric is used to evaluate the performance of recommendation methods in the end ${ }^{(8)}$. The F1-metric can balance the tradeoff between precision and recall. It is defined as in Eq. (12).

$$
F 1=\frac{2 \times \text { precision } \times \text { recall }}{\text { precision }+ \text { recall }}
$$

\subsection{Experiment results}

In Fig. 2, the performance of recommendation methods based on follow influence are compared, including PFI, IS, PFI+IS, PFI+RFI, and FI. The performance of IS method is lower than that of PFI, but the performance of PFI+IS and PFI+RFI are better than PFI. Thus, the IS and RFI can really improve the performance of the 
recommendation. Moreover, the performance of PFI+RFI is better than PFI+IS. It indicates that the review influence has more impact on the recommendation performance than the interaction strength. The FI method which takes PFI, IS and RFI into account has the best performance.

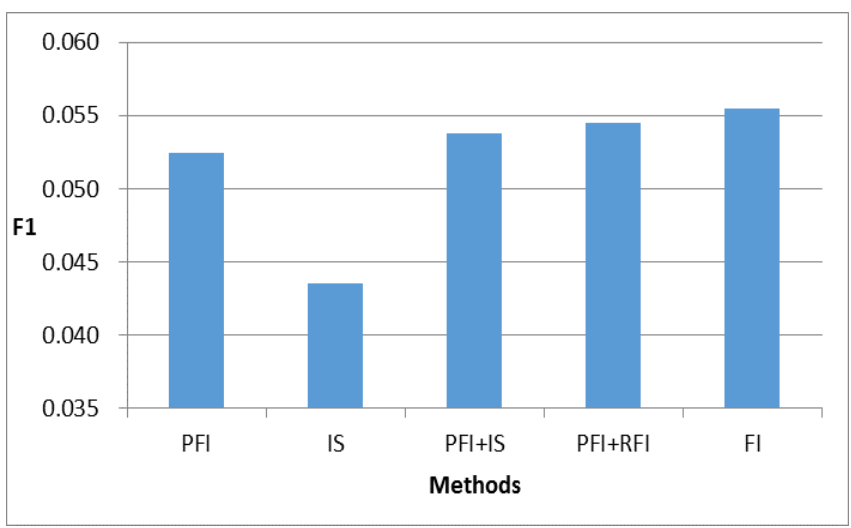

Fig. 1. The F1-metric with different methods based on the follow influence.

Finally, four recommendation methods, including UCF, II, FI and II+FI, are compared in Fig. 3. UCF method has the worst performance than other methods. It means that our proposed methods which makes recommendation based on interest influence or follow influence are better than the traditional recommendation method. In addition, FI method is better than II, so that the influence from followees is more effective than the influence from the similar users. Moreover, the proposed method II+FI which combines interest influence and follow influence outperforms other methods. When recommending Top-40 cosmetics, it has the highest F1 value than other methods. Therefore, recommendation based on different influences with personalized weights can improve the efficiency and accuracy of recommendation.

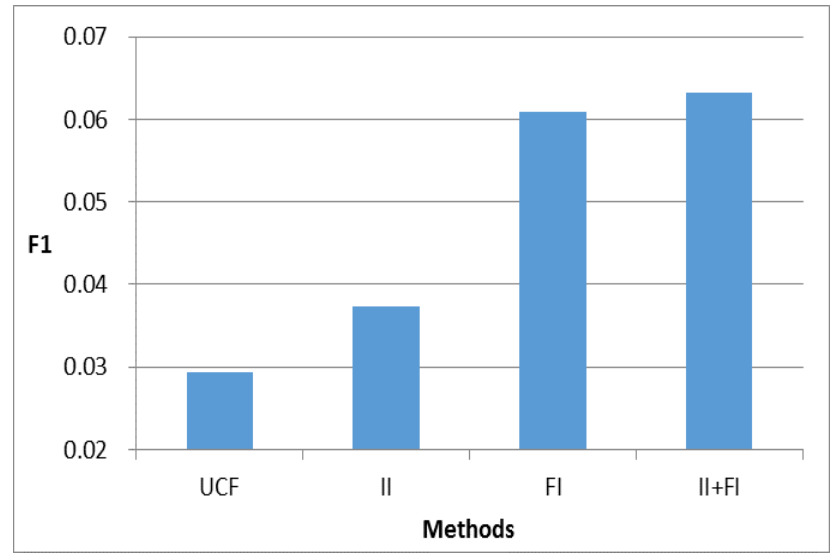

Fig. 2. The F1-metric with different recommendation methods.

\section{Conclusions}

In this work, we proposed a novel recommendation method based on two different types of influences, including the interest influence, follow influence and personalized weights for each influence, to recommend the cosmetics in a cosmetic-sharing social network website. For the follow influence, it consists of purchase-follow influence, interaction strength, and review influence. Besides, the time factor in follow influence is also considered for measuring the influence more precisely. The experimental results show that the time factor, the interaction strength and review influence can enhance the performance of recommendation. In addition, with combining the scores and personalized weights of two influences, it has the better performance than traditional method and other methods. Therefore, analyzing the interest and follow influences on a social network is useful in making recommendations. It also enhance the quality of social recommendations.

\section{Acknowledgment}

This research was supported by the Ministry of Science and Technology of Taiwan under the grant MOST 104-2410-H-033-027.

\section{References}

(1) J. Weng, C. Miao, and A. Goh, "Improving collaborative filtering with trust-based metrics," in Proceedings of the 2006 ACM Symposium on Applied Computing, Dijon, France, pp. 1860-1864, 2006.

(2) P. Massa, and P. Avesani, "Trust-aware recommender systems," in Proceedings of the 2007 ACM Conference on Recommender Systems, Minneapolis, MN, USA, pp. 17-24, 2007.

(3) Y.-M. Li, C.-H. Lin, and C.-Y. Lai, "Identifying influential reviewers for word-of-mouth marketing," Electronic Commerce Research and Applications, vol. 9, no. 4, pp. 294-304, 2010.

(4) P. Cui, F. Wang, S. Liu, M. Ou, S. Yang, and L. Sun, "Who should share what?: item-level social influence prediction for users and posts ranking," in Proceedings of the 34th International ACM SIGIR Conference on Research and Development in Information Retrieval, Beijing, China, pp. 185-194, 2011.

(5) J. Huang, X.-Q. Cheng, J. Guo, H.-W. Shen, and K. 
Yang, "Social recommendation with interpersonal influence,” in Proceedings of the 2010 Conference on ECAI 2010: 19th European Conference on Artificial Intelligence, pp. 601-606, 2010.

(6) K. Xu, X. Guo, J. Li, R. Y. K. Lau, and S. S. Y. Liao, "Discovering target groups in social networking sites: An effective method for maximizing joint influential power," Electronic Commerce Research and Applications, vol. 11, no. 4, pp. 318-334, 2012.

(7) D. Billsus, and M. J. Pazzani, "Learning collaborative information filters," in Proceedings of the Fifteenth International Conference on Machine Learning, pp. 46-54, 1998.

(8) B. Sarwar, G. Karypis, J. Konstan, and J. Riedl, "Analysis of recommendation algorithms for e-commerce," in Proceedings of the 2nd ACM Conference on Electronic Commerce, Minneapolis, Minnesota, United States, pp. 158-167, 2000. 